\title{
Reliability Analysis of the CAM on the Drive Gear Plate of D Type Knotter
}

\author{
Haitang Cen, Peiwen Li*, Ruitao Wei, and Xueke Si \\ School of Mechanical Engineering, Inner Mongolia University of Technology, Hohhot, 010000, China
}

\begin{abstract}
Cams of drive gear plates are key components of D type knotters. Wear is the main failure mode of cams. Accordingly, this paper studies the relationship between cams' wear and the reliability of a knot that is equipped in a D type knotter. Based on the theoretical and motional wear analysis of the cam mechanism assembled in a drive gear plate of a D type knotter, the mathematical relationship between cam wear and the displacement reliability of the cam follower is established. Moreover, the displacement reliability of the cam follower is calculated by joint simulation of the ADAMS virtual prototype and ANSYS. The following conclusions are reached after a considerable number of simulations: (1) The cam mechanism works at the stable wear stage at the time of design life. (2) The D type knotter will be unable to successfully tie knots anymore when the wear volume of the cam and contact surface of the follower reaches $2.3 \mathrm{~mm}$ (the cam follower displacement reliability is 0.9463 ). (3) $99 \%$ displacement reliability of the cam follower, at a workload of 20000 bales, requires the mean wear of the cam contact surface to be less than $0.87 \mathrm{~mm}$. A further conclusion is that maintenance and replacement measures are suggested to be taken at a workload of 20000 bales, to ensure that the displacement reliability of cam follower is more than $99 \%$ and the D type knot is workable.
\end{abstract}

Keywords: D type knotter; drive gear plate; cam; wear and tear; displacement reliability

(Submitted on September 7, 2018; Revised on October 8, 2018; Accepted on November 21, 2018)

C 2019 Totem Publisher, Inc. All rights reserved.

\section{Introduction}

The D type knot is one of the core components of the bundling machine [1]. The wear of the cam on the drive gear plate causes low binding rate, low reliability, and insufficient lifetime. The certifiable bundling rate of D type knots made in Germany and the USA is $99 \%$ during their designed life, even though the wear rate of cams on drive gear plates is also relatively high [2-3]. At present, how to improve the service life of D type knotters has become the bottleneck in reducing the price of baling machines. Therefore, aiming at addressing the wear problem of the cams of driving gear plates, this paper analyzed the knot failure and calculated its reliability so as to find the best maintenance strategy. The analysis is proven to be a serviceable process for the reliability estimation of D knotting machine.

\section{D-Knotter Overview}

A drive gear plate is an important part of the D type knotting machine. It provides power to the whole knotting machine, whose quality affects the efficiency of the entire baler knotting. It comprises one input shaft and three output lines for power transition. Output lines are designed to drive the other parts of the knot device movement by gear meshing and the cam mechanism. The outer rack has seven teeth, is evenly distributed on the axis of the coiling disc, and meshes with the incomplete gear mounted on the knot mouth, providing power output for the knotter jaw. The inner rack also has seven teeth, is a variant tooth that meshes with the incomplete gear mounted on the worm, and provides power output for the clip rope disc. The cam mechanism provides the power to diastasis the knot and cut rope for the off rope rod. There is a fixed phase difference between the two rack and the cam, and the phase difference determines the time sequence related to the cut rope and takes off the knot action after the knot mouth action [4-6]. The running time is short, fast, and highly precise, and the trajectory of the gear is also precise [7-9]. A new drive gear plate structure is shown in Figure 1.

* Corresponding author.

E-mail address: 1139421147@qq.com 
Figure 2 shows the wear condition of a D type knotter drive gear plate working 20 thousand times without maintenance. Because of the contact between the off rope rod and the knotter jaw, the stress on the contact surface of the cam and the cam follower increases, causing serious wear and tear. Moreover, owing to no maintenance, the assembly accuracy of the cam and the follower decreases; as a result, displacement reliability will decrease, then transmission error increases, wear aggravates, and vicious circle finally occurs, resulting in D knot knotting failure. Therefore, this paper carried out the movement reliability analysis and established mathematical models for calculating the relationship between the wear of the cam and the reliability of the cam motion transmission. The analyzed lifetime of the D type knotter will be the original input knowledge for the optimal maintenance strategy.

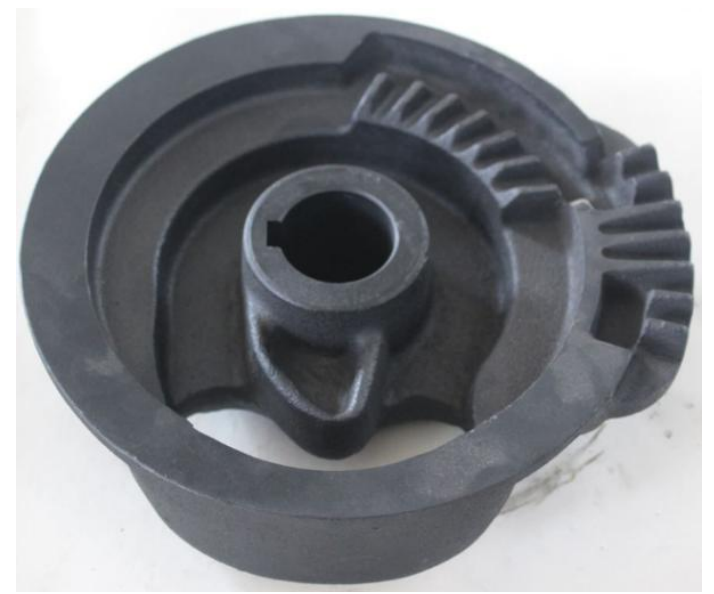

Figure 1. D type knotter driver gear plate

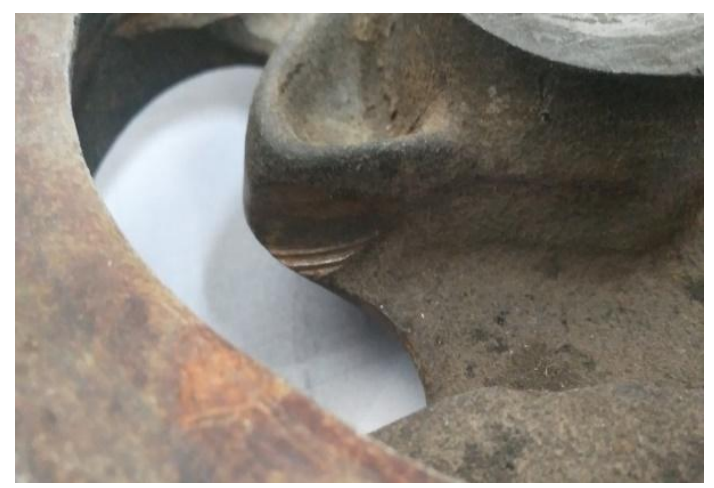

Figure 2. The cam wear diagram on the drive gear plate

\section{Failure Type and Failure Mechanism of Knotter}

A knotter bears a variety of twisting, shear, cutting, pressing, and other complex forces, coupled with knotting in the field. Harsh working environments make damages of the knotter a frequent occurrence. Through the statistics of the working environment, the force, and the actual operation of the baler, five main failure modes are found: clamp rope failure, rope around failure, cut rope failure, upper claw failure, and rope buckle failure. Actual working condition through knotter shows that the drive gear, frame, casting off rope lever, knotting mouth, clamping rope mechanism, bevel gear, and cutting of the rope knife are the main factors that cause failures. The failure of the gear plate and the knotter frame are the most important factors for knotter failure.

\subsection{Theoretical Analysis of Wear}

Wear is a phenomenon of micro loss of surface material caused by friction. Wear is generally divided into abrasive wear, fatigue wear, adhesive wear, oxidation wear, fretting wear, and selective migration. The wear of the cam of the D type knotter is mainly caused by fatigue wear. This paper discusses the influence of cam wear amount on cam output motion parameters without considering the original error of the cam profile and deduces the mathematical relationship between wear volume and output motion parameters. Generally, wear can be divided into three stages: running-in stage, stable stage, and accelerated wear stage, as shown in Figure 3. 
Figure 4 shows the changing law of wear velocity. The crests on the friction surface formed in the manufacturing process are easily abraded in the running-in stage, which results in high initial wear speed, and the curve of wear velocity in this stage is concave. The wear rate tends to be constant after the crest is polished. After that the contact surface gap exceeds the allowable range, which causes a sharp increase in dynamic load, the wear rate and wear suddenly increase to form an accelerated wear and tear. Obviously, the normal work should be in the stable stage and only a specified amount of wear is allowed in the contact surface. If it is out of the specified range, the mechanism's motion reliability will be affected. The wear amount and wear rate are described mathematically as follows.

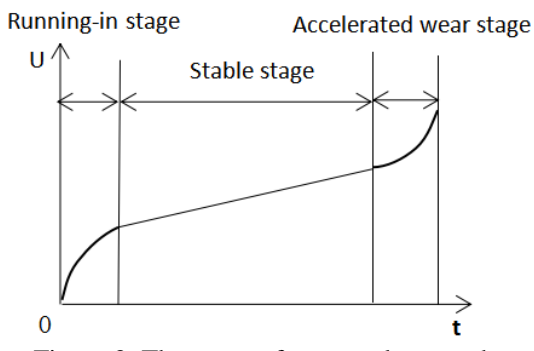

Figure 3. The curve of wear and tear path

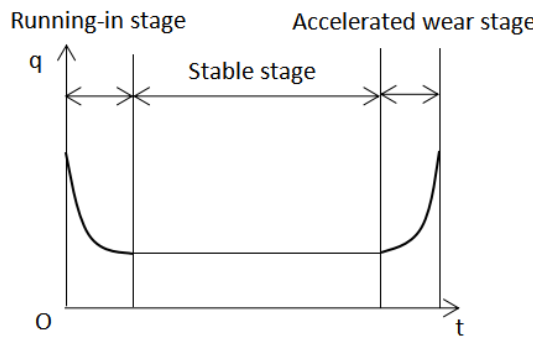

Figure 4. The curve of wear velocity changes

1) Running-in stage

Since the exact description of the wear curve is difficult and unrealistic, a similar curve is instead used in this paper. The wear speed of the running-in stage varies from fast to slow, assuming the wear speed follows a uniform deceleration. The relationship between wear rate and time is modelled as Equation (1).

$$
q_{1}=q_{0}-\gamma_{1} t
$$

Where $q_{1}$ is the wear rate at time $t, q_{0}$ is the initial wear speed, and $\gamma_{1}$ is the changing rate of the speed, which is a random constant greater than zero.

The relationship between the wear amount $U$ and time $t$ can be obtained by integrating Equation (1), shown in Equation (2).

$$
U_{1}=\int_{0}^{t} q_{1} d t=\int_{0}^{t}\left(q_{0}-\gamma t\right) \mathrm{d} t=q_{0} t-\frac{1}{2} \gamma_{1} t^{2}
$$

Equations (1) and (2) represent the functional relationship between wear amount and time in the stage of running-in.

2) Stable stage

The wear rate in the stable stage is a constant value, shown in Equation (3):

$$
q_{2}=q_{c}
$$

By integrating Equation (3), the relationship between wear amount and time in this stage can be reached, shown in Equation (4).

$$
U_{2}=q_{c} t
$$

3) Accelerated wear stage

Wear speed is accelerated in this stage, and the relationship between the wear speed and the time can be found in Equation (5).

$$
q_{3}=q_{0}+\gamma_{2} t
$$

Where $q_{3}$ is the wear rate at time $t, q_{0}$ is the initial wear speed, and $\gamma_{2}$ is the changing rate of speed and a constant greater than zero. 
The relationship between the wear amount $U$ and time $t$ can be obtained by integrating Equation (5), shown in Equation (6).

$$
U_{3}=\int_{0}^{t} q_{3} d t=\int_{0}^{t}\left(q_{0}+\gamma t\right) \mathrm{d} t=q_{0} t+\frac{1}{2} \gamma t^{2}
$$

Equations (5) and (6) represent the functional relationship between wear amount and time in the stage of accelerated wear.

\subsection{The Motion Analysis of the Cam Mechanism on the Drive Gear Plate}

The coordinate diagram of cam mechanism on drive gear plate is established in Figure 5. Mathematical expressions for cam follower displacements are modeled by polar form. The polar coordinates of the point $\mathrm{M}$ at the center of the cam follower is expressed in Equation (7).

$$
\begin{aligned}
& \rho=\sqrt{\left(s+s_{0}\right)^{2}+e^{2}} \\
& \psi=\theta-\phi+\beta \\
& \tan \beta=\frac{s+s_{0}}{e} \\
& s_{0}=\sqrt{\left(r_{b}+r_{r}\right)^{2}-e^{2}}
\end{aligned}
$$

Where $\rho$ is the radial function, $\psi$ is the radial angle, $\phi$ is the angle of cam rotation, $\theta$ is the angle between the linear $O P_{0}$ and the $\chi$ axis, $s$ is the output displacement of the cam follower, $r_{b}$ is the outer arc radius of the cam, $r_{r}$ is the radius of the cam follower, and $e$ is the axial hole radius of the camshaft $[1,10]$.

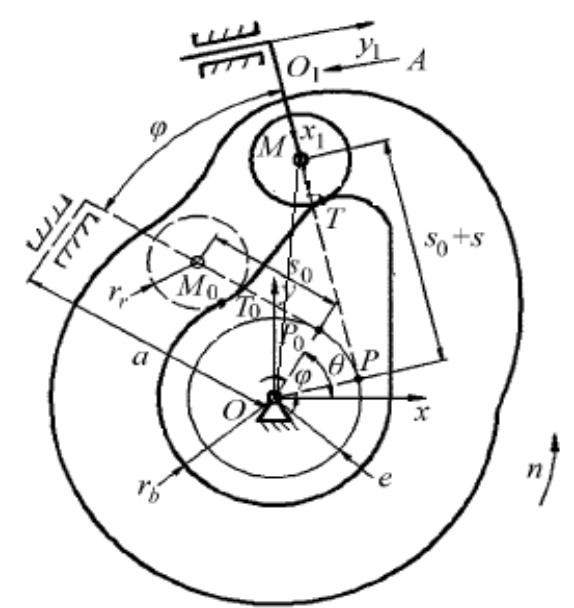

Figure 5. The coordinate diagram of cam mechanism on drive gear plate

The cam profile curve equation is calculated in Equation (8).

$$
\begin{aligned}
& f(\rho, \psi)=0 \\
& \rho=\rho(\psi) \\
& \psi=\psi[\phi(t)]
\end{aligned}
$$

Where $\phi$ is the angle of the cam rotation, and it is a function of time.

Combining Equations (7) and (8), we get the relationship between $s$ and $\rho$.

$$
s=f\left[\rho(\psi), r_{0}, e\right]
$$


By combining Equations (7) and (9), a mathematical relationship between the output displacement of the cam follower, the cam profile parameters, and the basic parameters of the cam can be established as Equation (10).

$$
\begin{aligned}
& s=\sqrt{\rho^{2}-e^{2}}-s_{0} \\
& s_{0}=\sqrt{r_{0}^{2}-e^{2}} \\
& r_{0}=r_{b}+r_{r}
\end{aligned}
$$

Where $\phi$ is a function of the time, and $r_{0}$ and $e$ are constant.

\subsection{Establishing Reliability Calculation Model of Cam Motion Containing Wear}

The cam mechanism is connected with the high side. Wear occurs mainly in the cam's contour line. Wear affects the radial error only. Relationships between wear and cam profile error and that between wear and cam follower output error are established.

1) Establishing the relationship between wear and cam profile error.

The wear relation in three stages has been established in the previous study, shown in Equation (11).

$$
\begin{aligned}
& U_{1}=q_{0} t-\frac{1}{2} \gamma t^{2} \\
& U_{2}=q_{c} t \\
& U_{3}=q_{0} t+\frac{1}{2} \gamma t^{2}
\end{aligned}
$$

At a certain angle, the total error of the CAM profile is equal to the sum of the contour error without wearing and the wear amount, which is modelled in Equation (12).

$$
\Delta=\Delta^{*}+U
$$

Where $\Delta$ is the total error of cam profile, $\Delta^{*}$ is the cam profile error without wear, and $U$ is the total amount of wear.

The total cam profile error for the three wear stages can be calculated by combining Equations (11) and (12).

Running-in stage:

$$
\Delta_{1}=\Delta_{1}^{*}+\left(q_{0} t-\gamma t^{2}\right)
$$

Stable stage:

$$
\Delta_{2}=\Delta_{2}^{*}+q_{c} t
$$

Accelerated wear stage:

$$
\Delta_{3}=\Delta_{3}^{*}+\left(q_{0} t+\gamma t^{2} / 2\right)
$$

According to the statistics, the mean parameters of mean and variance in the three stages are calculated in Equations (16)-(21).

$$
\begin{gathered}
E\left(\Delta_{1}\right)=E\left(\Delta_{1}^{*}\right)+E\left(q_{0}\right) t-E(\gamma) t^{2} / 2 \\
=\mu_{\Delta_{1}^{*}}+\mu_{q_{0}} t-\mu_{\gamma} t^{2} / 2
\end{gathered}
$$




$$
\begin{gathered}
E\left(\Delta_{2}\right)=E\left(\Delta_{2}^{*}\right)+E\left(q_{c}\right) t \\
=\mu_{\Delta_{1}^{*}}+\mu_{q_{c}} t \\
E\left(\Delta_{3}\right)=E\left(\Delta_{3}^{*}\right)+E\left(q_{0}\right) t+E(\gamma) t^{2} / 2 \\
=\mu_{\Delta_{3}^{*}}+\mu_{q_{0}} t+\mu_{\gamma} t^{2} / 2 \\
\sigma_{\Delta_{1}}^{2}=\sigma_{\Delta_{1}^{*}}^{2}+\sigma_{q_{0}}^{2} t^{2}+\sigma_{\gamma}^{2} t^{4} / 4 \\
\sigma_{\Delta_{2}}^{2}=\sigma_{\Delta_{2}^{*}}^{2}+\sigma_{q_{c}}^{2} t^{2} \\
\sigma_{\Delta_{3}}^{2}=\sigma_{\Delta_{3}^{*}}^{2}+\sigma_{q_{0}}^{2} t^{2}+\sigma_{\gamma}^{2} t^{4} / 4
\end{gathered}
$$

The expected value of the total profile error of the cam changes with time in different wear stages. The longer the time, the greater the error mean and greater the influence on the output motion. Hence, the characteristic values of the profile error of the cam in the three wear stages are obtained.

2) Establishing the relationship between wear and output error of the cam follower:

Symbol * represents the ideal value, and the error is expressed by $\Delta$. Based on this, Equation (22) is obtained from Equation (9). Equation (22) at the ideal value is extended by first-order Taylor series expansion, shown in Equation (23).

$$
\begin{gathered}
s=s^{*}+\Delta s=F\left(\rho^{*}+\Delta \rho, r_{0}^{*}+\Delta r_{0}, e^{*}+\Delta e\right) \\
\Delta s=\frac{d s}{d \rho} \Delta \rho+\frac{d s}{d r_{0}} \Delta r_{0}+\frac{d s}{d e} \Delta e
\end{gathered}
$$

The mean and variance of the cam follower displacements are calculated in Equation (24) under the circumstance of mean of are respectively $\mu_{\rho}, \mu_{r_{0}}, \mu_{e}$, and the standard deviations are $\sigma_{\rho}, \sigma_{r_{0}}, \sigma_{e}$. Wear only affects the profile error of the cam, and the eigenvalues of the cam profile error in the three wear stages are shown in Equation (25).

$$
\begin{gathered}
\mu_{\mathrm{s}}=\frac{d s}{d \rho} \mu_{\rho}+\frac{d s}{d r_{0}} \mu_{r_{0}}+\frac{d s}{d e} \mu_{e} \\
\sigma_{s}^{2}=\left(\frac{d s}{d \rho}\right)^{2} \sigma_{\rho}^{2}+\left(\frac{d s}{d r_{0}}\right)^{2} \sigma_{r_{0}}^{2}+\left(\frac{d s}{d e}\right)^{2} \sigma_{e}^{2} \\
\mu_{\mathrm{p} z_{1}}=\mu_{p}+\mu_{q_{0}} t-\mu_{\gamma} t^{2} / 2 \\
\mu_{\mathrm{p} z_{2}}=\mu_{p}+\mu_{q_{c}} \\
\mu_{p z_{3}}=\mu_{p}+\mu_{q_{0}} t+\mu_{\gamma} t^{2} / 2 \\
\sigma_{p \sigma_{1}}^{2}=\sigma_{p}^{2}+\sigma_{q_{0}}^{2} t^{2}+\sigma_{\gamma}^{2} t^{4} / 4 \\
\sigma_{p \sigma_{2}}^{2}=\sigma_{p}^{2}+\sigma_{q_{c}}^{2} t^{2} \\
\sigma_{p \sigma_{3}}^{2}=\sigma_{p}^{2}+\sigma_{q_{0}}^{2} t^{2}+\sigma_{\gamma}^{2} t^{4} / 4
\end{gathered}
$$

Where $\mu_{q_{0}}$ and $\mu_{q_{c}}$ are the mean values of the initial speed of wear, $\sigma_{q_{c}}^{2}$ and $\sigma_{q_{0}}^{2}$ are the variances of wear speed, $\mu_{\gamma}$ is the mean of the rate of velocity change, and $\sigma_{\gamma}^{2}$ is the variance of the rate of velocity change. 
$\mu_{p z_{1}}, \mu_{p z_{2}}, \mu_{p z_{3}}, \sigma_{p \sigma_{1}}^{2}, \sigma_{p \sigma_{2}}^{2}$, and $\sigma_{p \sigma_{3}}^{2}$ are used to consider the wear of $\mu_{p}$ and $\sigma_{p}^{2}$, and then three wear stages of the cam displacement output parameter error relationships (shown in Equations (26)-(28)) can be obtained by Equation (24).

Running-in stage:

$$
\begin{aligned}
\mu_{\mathrm{s}} & =\frac{d s}{d p} \mu_{p z_{1}}+\frac{d s}{d r_{0}} \mu_{r_{0}}+\frac{d s}{d e} \mu_{e} \\
& =\frac{d s}{d p}\left(\mu_{p}+\mu_{q_{0}} t-\mu_{\gamma} t^{2} / 2\right)+\frac{d s}{d r_{0}} \mu_{r_{0}}+\frac{d s}{d e} \mu_{e} \\
\sigma_{s}^{2} & =\left(\frac{d s}{d p}\right)^{2}\left(\sigma_{p}^{2}+\sigma_{q_{0}}^{2} t^{2}+\sigma_{\gamma}^{2} t^{4} / 4\right)+\left(\frac{d s}{d r_{0}}\right)^{2} \sigma_{r_{0}}^{2}+\left(\frac{d s}{d e}\right)^{2} \sigma_{e}^{2}
\end{aligned}
$$

Stable stage:

$$
\begin{aligned}
\mu_{\mathrm{s}} & =\frac{d s}{d p} \mu_{p z_{1}}+\frac{d s}{d r_{0}} \mu_{r_{0}}+\frac{d s}{d e} \mu_{e} \\
& =\frac{d s}{d p}\left(\mu_{p}+\mu_{q_{c}} t\right)+\frac{d s}{d r_{0}} \mu_{r_{0}}+\frac{d s}{d e} \mu_{e} \\
\sigma_{s}^{2} & =\left(\frac{d s}{d p}\right)^{2}\left(\sigma_{p}^{2}+\sigma_{q_{c}}^{2} t^{2}\right)+\left(\frac{d s}{d r_{0}}\right)^{2} \sigma_{r_{0}}^{2}+\left(\frac{d s}{d e}\right)^{2} \sigma_{e}^{2}
\end{aligned}
$$

Accelerated wear stage:

$$
\begin{aligned}
\mu_{\mathrm{s}} & =\frac{d s}{d p} \mu_{p z_{1}}+\frac{d s}{d r_{0}} \mu_{r_{0}}+\frac{d s}{d e} \mu_{e} \\
& =\frac{d s}{d p}\left(\mu_{p}+\mu_{q_{0}} t+\mu_{\gamma} t^{2} / 2\right)+\frac{d s}{d r_{0}} \mu_{r_{0}}+\frac{d s}{d e} \mu_{e} \\
\sigma_{s}^{2} & =\left(\frac{d s}{d p}\right)^{2}\left(\sigma_{p}^{2}+\sigma_{q_{0}}^{2} t^{2}+\sigma_{\gamma}^{2} t^{4} / 4\right)+\left(\frac{d s}{d r_{0}}\right)^{2} \sigma_{r_{0}}^{2}+\left(\frac{d s}{d e}\right)^{2} \sigma_{e}^{2}
\end{aligned}
$$

Wear affects the mean and variance of the output displacement error of the cam. The longer the time, the greater the influence will be. The characteristic value of the cam profile wear speed and the allowable limit error characteristic value of the cam follower by motion simulation of the cam wear is required for calculating the displacement reliability of the cam including wear.

\subsection{Motion Simulation of Cam Wear}

First, the finite element model of the drive gear plate of d-type knotter is established, and then it is imported into the ADAMS. A virtual experimental prototype for the cam and follower engagement is established, as shown in Figure 6.

The boundary conditions and initial loads are defined according to the actual working parameters of the D type knotter. At the same time, the speed of the drive gear plate and the torque of the spindle are inputs, and then the motion simulation is carried out.

We extract the simulation results of two aspects in the process of simulation. On one hand, we analyze the D type knotter successful knot boundary conditions to obtain the cam follower displacement allowable range and limit values. After many simulation results, the cam-follower displacement allowable limit error characteristic value $\left(\mu_{0}, \sigma_{0}\right)$ is obtained. On the other hand, we obtain the stress load spectrum of the cam target contact surface. After obtaining the stress load spectrum of the contact surface of the cam, the stress damage analysis of the cam is carried out by ANSYS software to obtain the displacement damage amount and the cycle life. Then, we compare the displacement damage amount with the limit allowable wear amount. If the displacement damage amount is less than the allowable limit of the cam wear, we modify the 
cam finite element model according to the amount of damage. Then, we proceed to the next cycle of motion simulation until the displacement damage is greater than or equal to the limit of the cam, and then we stop the simulation. Concrete realization of the process is shown in Figure 8. In this way, we can get the maximum movement times before the failure of the cam and the maximum working life by multiple motion simulation. According to the maximum displacement damage, we can obtain the wear speed, and then we can get the characteristic value $\left(\mu_{q_{0}}, \sigma_{q_{0}}\right)$ of the cam profile wear speed according to the results of multiple simulation.

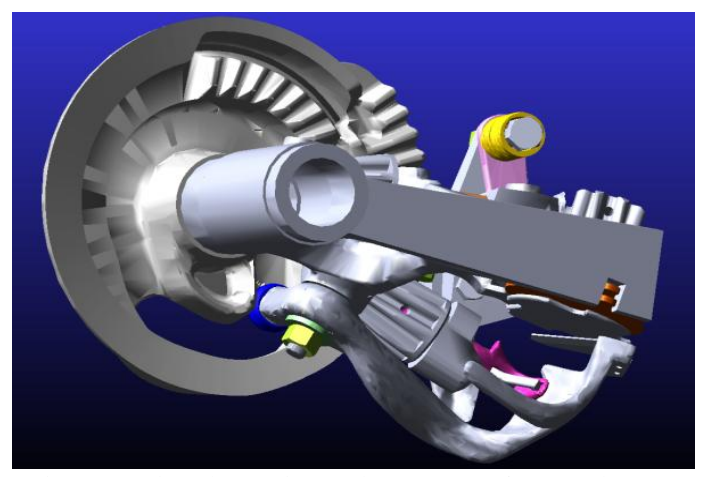

Figure 6. Virtual experimental prototype of D type knotter

Figure 7 shows the stress change versus time for the target contact surface without wear of the cam profile when driving the gear plate for two cycles in an ADAMS virtual prototype.

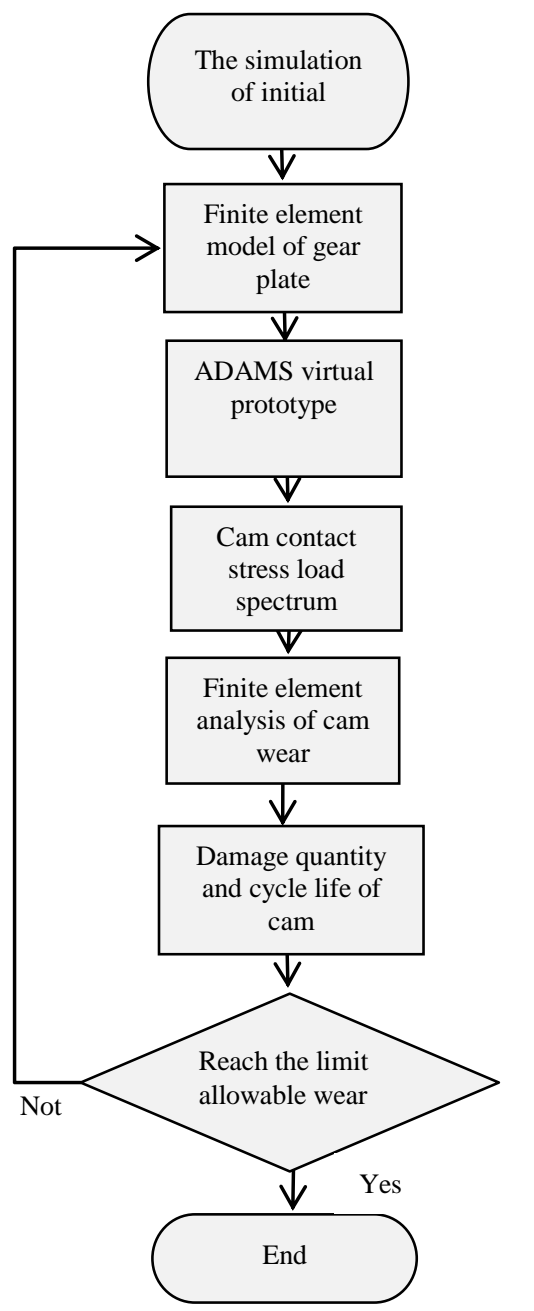

Figure 7. Virtual prototype simulation flow chart of cam 
As shown in Figure 8, the maximum stress that can be obtained in the measurement interface to the cam target contact surface occurs at $0.529 \mathrm{~s}$ and $1.19 \mathrm{~s}$, which are the times when the off rope rod takes off the knot. Meanwhile, due to the contact between the off rope rod and the knotter jaw, the stress between the cam and the cam follower contact surface suddenly increases (the maximum stress is up to $1179 \mathrm{~N}$ ). The maximum stress is loaded in a cyclic form to the target contact surface of the cam in ANSYS software. The cycle time of the applied load is the design life of the D type knotter. Then, according to the actual material properties and motion parameters of the cam, the static damage analysis of the cam is carried out, and the damage displacement and deformation cloud map of the cam is obtained as shown in Figure 9.

It can be seen from Figure 9 that the maximum displacement damage of the cam contact surface is $0.304 \mathrm{~mm}$. The D type knotter will fail when the displacement damage amount of the cam contact surface reaches $2.34 \mathrm{~mm}$. The stress of the cam contact surface increases to $6000 \mathrm{~N}$ at that time. It is known that when the displacement wear amount of the cam contact surface reaches $2.34 \mathrm{~mm}$, the D type knotter cannot be successfully knotted.

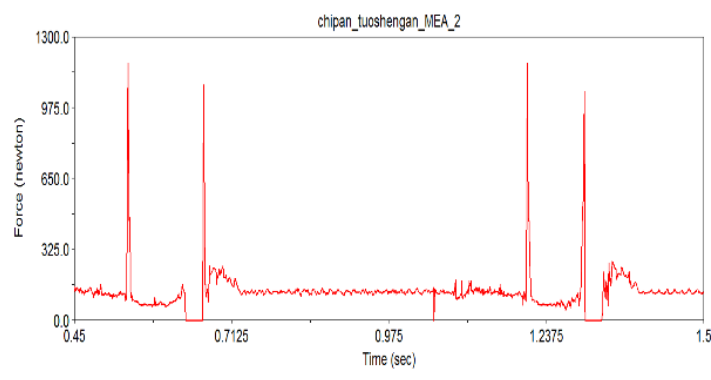

Figure 8. Virtual prototype simulation flow chart of cam

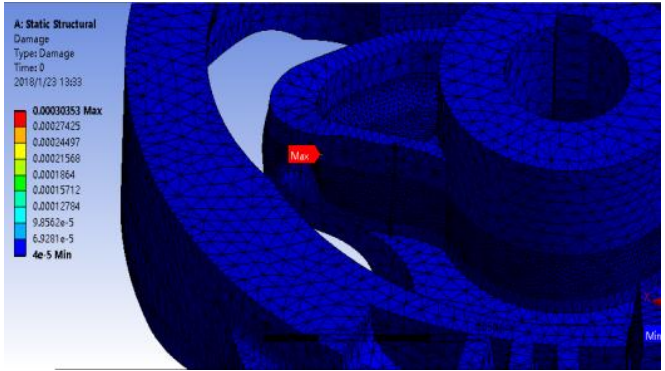

Figure 9. Virtual prototype simulation flowchart of cam

After multiple simulations, the D type knotting machine can successfully knot to the unsuccessful knotting. The limit wear diagram of the cam contact surface is obtained as shown in Figure 10.

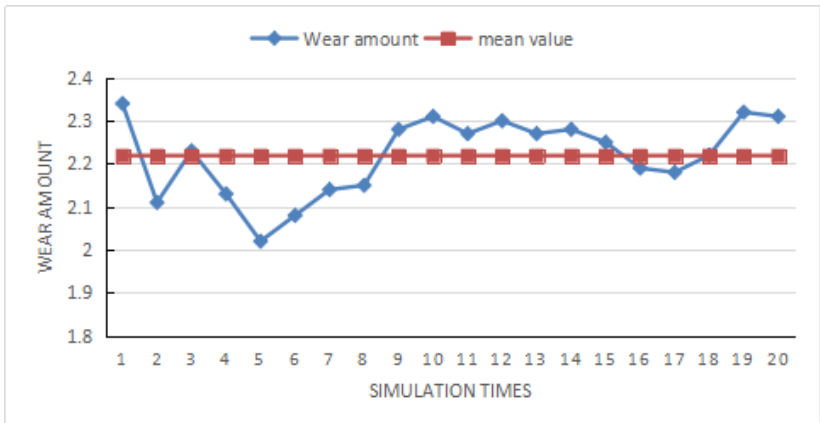

Figure 10. Limit displacement wear of cam contact surface

From the simulation results shown above, the displacement allowable limit error eigenvalue of the cam follower is obtained according to the probability formula $\left(\mu_{0}, \sigma_{0}\right)=(2.219,0.09)$. According to the flowchart shown in Figure 7 , after 20 cycles of simulation, the wear displacement results are obtained, shown in Table 1 and Figure 11.

Table 1. Simulation results of cam wear displacement

\begin{tabular}{|c|c|c|c|}
\hline \multicolumn{5}{|c|}{ Table 1. Simulation results of cam wear displacement } \\
\hline Simulation time & Wear displacement & Simulation time & Wear displacement \\
\hline 1 & 0.304 & 11 & 0.864 \\
\hline 2 & 0.339 & 12 & 0.964 \\
\hline 3 & 0.376 & 13 & 1.076 \\
\hline 4 & 0.416 & 14 & 1.201 \\
\hline 5 & 0.46 & 15 & 1.34 \\
\hline 6 & 0.509 & 16 & 1.494 \\
\hline 7 & 0.564 & 17 & 1.664 \\
\hline 8 & 0.626 & 18 & 1.851 \\
\hline 9 & 0.696 & 19 & 2.056 \\
\hline 10 & 0.775 & 20 & 2.34 \\
\hline
\end{tabular}




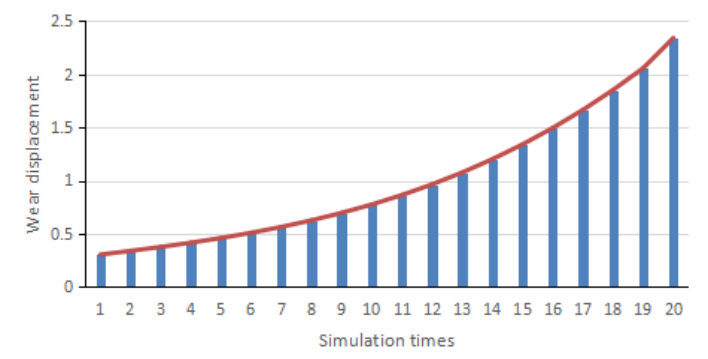

Figure 11. The curve of the wear displacement of the cam contact surface

According to the design service life of the $\mathrm{D}$ type knotter and the working cycle of the cam $T=0.67 \mathrm{~s}$, the total working time of the cam is calculated by $t=7.44 \mathrm{~h}$. Meanwhile, the wear speed of the cam contact surface can be calculated, shown in Table 2 and Figure 12.

Table 2. Simulation results of cam wear speed

\begin{tabular}{|c|c|c|c|}
\hline Simulation time & Wear speed & Simulation time & Wear speed \\
\hline 1 & 0.0408 & 11 & 0.1041 \\
\hline 2 & 0.0455 & 12 & 0.1161 \\
\hline 3 & 0.0505 & 13 & 0.1295 \\
\hline 4 & 0.0559 & 14 & 0.1446 \\
\hline 5 & 0.0618 & 15 & 0.1614 \\
\hline 6 & 0.0684 & 16 & 0.1801 \\
\hline 7 & 0.0758 & 17 & 0.2008 \\
\hline 8 & 0.0841 & 18 & 0.2236 \\
\hline 9 & 0.0935 & 19 & 0.2487 \\
\hline 10 & 0.0408 & 20 & 0.2763 \\
\hline
\end{tabular}

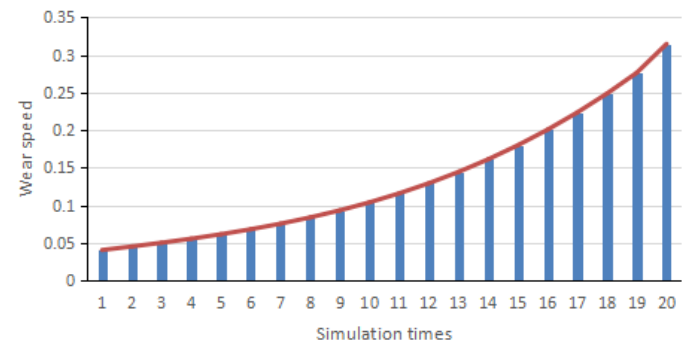

Figure 12. The curve of the wear speed of the cam contact surface

According to the results shown above, the eigenvalues of the wear speed of the cam contact surface can be calculated according to the probability formula: $\left(\mu_{q_{0}}, \sigma_{q_{0}}\right)=(0.134,0.083)$. According to the simulation results and the actual size parameters and motion parameters of the cam, the reliability of cam follower displacement, speed, and acceleration can be calculated in the three wear stages when the running time reaches the design life.

\subsection{Calculation of the Displacement Reliability of the Cam on the Drive Gear Plate in the Stable Stage}

According to Equation 10 and the mathematical model of the cam on the drive gear plate, Equations (29) and (30) are obvious.

$$
\begin{aligned}
& \frac{\mathrm{d} s}{\mathrm{~d} \rho}=\frac{\rho}{\sqrt{\rho^{2}-e^{2}}} \\
& \frac{\mathrm{d} s}{\mathrm{~d} r_{0}}=\frac{\mathrm{d} s}{\mathrm{~d} s_{0}} \frac{\mathrm{d} s_{0}}{\mathrm{~d} r_{0}}=-\frac{r_{0}}{\sqrt{r_{0}^{2}-e^{2}}} \\
& \frac{\mathrm{d} s}{\mathrm{~d} e}=\frac{-e}{\sqrt{\rho^{2}-e^{2}}}+\frac{e}{\sqrt{r_{0}^{2}-e^{2}}}
\end{aligned}
$$




$$
\begin{aligned}
& s=\frac{2 h}{\phi_{1}^{2}} \alpha^{2} \\
& \rho=\sqrt{\left(s+s_{0}\right)^{2}+e^{2}}
\end{aligned}
$$

Where $h$ is the distance of run in the cam follower, $\alpha$ is the angle of the cam rotation, and $\phi_{1}$ is the push travel angle of the cam [11-13].

In the stable wear stage, the basic cam size parameters and motion parameters are shown in Table 3 [14-17].

Table 3. The basic parameters of the cam on the drive gear plate

\begin{tabular}{|c|c|c|c|}
\hline The push travel angle $\phi_{1}:$ & $0 \sim 63.170$ & The radius of the cam follower & $1.25 \mathrm{~cm}$ \\
\hline The return angle $\phi_{2}:$ & $63.170 \sim 123.170$ & CAM rotation speed $n:$ & $90 \mathrm{r} / \mathrm{min}$ \\
\hline The distance of run in cam follower $h:$ & $2 \mathrm{~cm}$ & CAM angular velocity $\omega:$ & $3 \pi \mathrm{rad} / \mathrm{s}$ \\
\hline The radius of camshaft $e:$ & $2.8 \mathrm{~cm}$ & Design service life $t:$ & 40000 time \\
\hline The Outer arc radius of cam $r_{b}:$ & $4.2 \mathrm{~cm}$ & & \\
\hline
\end{tabular}

Substituting the cam parameters into Equations (29) and (30), the following results can be obtained:

$$
s=1.181, \rho=6.495, \frac{\mathrm{d} s}{\mathrm{~d} \rho}=1.108, \frac{\mathrm{d} s}{\mathrm{~d} r_{0}}=-1.166, \frac{\mathrm{d} s}{\mathrm{~d} e}=0.121
$$

For the cam in the steady wear stage, according to Equation (27) and Equation (31) can be obtained:

$$
\begin{aligned}
& \mu_{s}=\frac{\mathrm{d} s}{\mathrm{~d} \rho}\left(\mu_{p}+\mu_{q_{0}} t\right)+\frac{\mathrm{d} s}{\mathrm{~d} r_{0}} \mu_{r_{0}}+\frac{\mathrm{d} s}{\mathrm{~d} e} \mu_{e} \\
& \sigma_{s}^{2}=\left(\frac{\mathrm{d} s}{\mathrm{~d} \rho}\right)^{2}\left(\sigma_{\rho}^{2}+\sigma_{q_{c}}^{2} t^{2}\right)+\left(\frac{\mathrm{d} s}{\mathrm{~d} r_{0}}\right)^{2} \sigma_{r_{0}}^{2}+\left(\frac{\mathrm{d} s}{\mathrm{~d} e}\right)^{2} \sigma_{e}^{2}
\end{aligned}
$$

The original profile error of the cam is not considered. Therefore, $\mu_{\rho}=\mu_{r_{0}}=\mu_{e}=0, \sigma_{\rho}=\sigma_{r_{0}}=\sigma_{e}=0$. The above data is substituted into Equation (31), and the following results are obtained: $\mu_{\mathrm{s}}=1.105, \sigma_{s}^{2}=0.468$. The displacement error data of cam wear obeys the normal distribution of $\mu=2.219$. When the displacement error of the cam wear is greater than $2.219 \mathrm{~mm}$, the D type knotter cannot be successfully knotted. According to the normal distribution, the statistical formula is:

$$
\begin{aligned}
& R=\Phi(\beta) \\
& \beta=\frac{\mu_{0}-\mu_{S}}{\sqrt{\sigma_{0}^{2}+\sigma_{S}^{2}}}
\end{aligned}
$$

Substituting the data into Equation (32), conclusions that $\beta=0$ and the reliability is 0.9463 are reached. When the average error of CAM wear is $0.87 \mathrm{~mm}$, the reliability is 0.9909 and the CAM been run to an average of 2230 times, namely the design of the CAM is about half the service life.

\section{Conclusions}

The cam mechanism equipped in the drive gear plate of the $\mathrm{D}$ type knotter under normal working condition works at a steady wear stage after the analysis. Without considering the original error of the cam profile, the cam and follower of the contact surface erosion rate will reach $2.3 \mathrm{~mm}$ at the D type knot's design life, and the cam follower displacement reliability is 0.9463 . The D type knotter will be unable to successfully knot when the wear is larger than $2.3 \mathrm{~mm}$. The conclusion that the displacement reliability of the cam follower is more than $99 \%$ requires the mean value of the displacement wear of the cam contact surface to be less than $0.87 \mathrm{~mm}$ after multiple simulation and calculation. Meanwhile, the average working time of the cam is about half of the designed service life. Therefore, it is recommended that the maintenance for the cam mechanism of the D type knotter should be taken after 20,000 bales to ensure that the reliability of the displacement of the 
cam follower is higher than $99 \%$ and the D type knotter can be successfully knotted.

\section{Acknowledgements}

This work is supported by the National Natural Science Foundation of China (No. 51365031) and the National Key Research and Development Project of China (No. 2016YFD0701700).

\section{References}

1. H. K. Chen, "Mechanical Foundation," China Labor Social Security Press, Beijing, 2007

2. L. J. Chen, C. li, and A. Q. Zhang, "Reconstruction and Motion Simulation of D-Type Knotting based on Reverse Engineering," Journal of Agricultural Mechanics, Vol. 45, No. 13, pp. 104-108, 2014

3. J. Dong and Y. Wen, "Rotational Swashplate Pulse Continuously Variable Transmission based on Helical Gear Axial Meshing Transmission," Chinese Journal of Mechanical Engineering, Vol. 25, No. 6, pp. 1138-1143, 2012

4. W. Huang, Z. Y. Zhang, and Z. Q. Liu, "Pro/Engineer Simulation and Action Function Analysis of D-Type Knotter," Research on Agricultural Mechanization, pp. 58-60, 2009

5. H. Li, J. He, and H. W. Li, "Study on the Spatial Parameters of the Knotting Machine of the Square Bundle Balere," Journal of Agricultural Machinery, Vol. 44, No. 8, pp. 9-105, 2013

6. H. Li, J. He, Q. J. Wang, H. W. Li, and R. R. Gautam, "Design and Experiment of D-Type Knots Knotters on Chinese Small Square Balers," Chinese Journal of Mechanical Engineering, Vol. 27, No. 1, pp. 154-165, 2014

7. H. Li, H. W. Li, and J. He, "Construction and Optimization of the Drive Plate for D-Type Knotting Machine with Square Straw Baler," Journal of Agricultural Mechanization, Vol. 26, No. 5, pp. 97-102, 2010

8. H. T. Li, Y. Xiong, L. J. Chen, S. Y. Zhang, X. Li, and L. J. Han, "Wear Analysis and Improved Design of Cutting Rope Tripping Mechanism," Journal of Agricultural Machinery, Vol. 46, No. 3, pp. 118-124, 2015

9. S. Li, "D-Type Knotted Rope Tied Knot Principle and Optimization Design," Ph.D. Jiangsu University, 2011

10. J. Lu, "Study on Structure and Parameter Optimization of the D-Type Knotter of Straw Baling Machine," Ph. D. Northeast Agricultural University, 2014

11. D. Z. Ren, C. G. Wang, and X. Y. Li, "Drive Tooth Plate Modal Analysis of D-Type Knotter," Agricultural Mechanization Research, pp. 58-65, 2014

12. G. Su, J. X. Shi, and J. Ge, "Measurement of the Space Angle of the Knotting Machine of a Baling Machine based on Reverse Engineering," Journal of Agricultural Machinery, Vol. 39, No. 6, pp. 81-83, 2008

13. Z. L. Sun, G. Z. Ji, Y. T. Yan, and Q. Yang, "Design and Analysis Technology of Mechanism Motion Reliability," National Defense Industry Press, Beijing, 2015

14. Q. H. Wan, K. Bu, Z. Y. Zhang, and B. S. Li, "Structure and Motion Analysis of D-Type Knotting Device," Agricultural Mechanization Research, pp. 17-19, 2009

15. J. R. Xu, "Research on CAD /CAM of Acceleration and Deceleration Cam Mechanism," Journal of Mechanical Engineering and Automation, No. 5, pp. 71-72, 2011

16. Y. Xiong, H. T. Li, S. Y. Zhang, L. J. Chen, X. Li, and L. J. Han, "Study on the Motion Law and Design Basis of a Knot Cutter to Cut the Rope Tripping Mechanism," Journal of Agricultural Mechanization Research, No. 7, pp. 113-118, 2015

17. J. J. Yin, S. li, and Y. M. li, "Simulation and Timing Analysis of D-Type Knotter and Its Auxiliary Mechanism," Journal of Agricultural Machinery, Vol. 42, No. 6, pp. 103-107, 2011 